\title{
Osserman Conditions in Lightlike Warped Product Geometry
}

\author{
Domitien Ndayirukiye ${ }^{*}$, Aboubacar Nibirantiza², Gilbert Nibaruta1, \\ Ménédore Karimumuryango ${ }^{3}$
}

\author{
${ }^{1}$ Département des Sciences Naturelles, Ecole Normale Supérieure, Bujumbura, Burundi \\ ${ }^{2}$ Département des Mathématiques, Institut de Pédagogie Appliquée, Université du Burundi, Bujumbura, Burundi \\ ${ }^{3}$ Institut des Statistiques Appliquées, Université du Burundi, Bujumbura, Burundi \\ Email: domitienndayi@yahoo.fr
}

How to cite this paper: Ndayirukiye, D., Nibirantiza, A., Nibaruta, G. and Karimumuryango, M. (2020) Osserman Conditions in Lightlike Warped Product Geometry. Journal of Applied Mathematics and Physics, 8, 585-596.

https://doi.org/10.4236/jamp.2020.84045

Received: February 11, 2020

Accepted: March 23, 2020

Published: March 26, 2020

Copyright $(02020$ by author(s) and Scientific Research Publishing Inc. This work is licensed under the Creative Commons Attribution International License (CC BY 4.0).

http://creativecommons.org/licenses/by/4.0/

\begin{abstract}
In this paper, we consider Osserman conditions on lightlike warped product (sub-)manifolds with respect to the Jacobi Operator. We define the Jacobi operator for lightlike warped product manifold and introduce a study of lightlike warped product Osserman manifolds. For the coisotropic case with totally degenerates first factor, we prove that this class consists of Einstein and locally Osserman lightlike warped product.
\end{abstract}

\section{Keywords}

Lightlike Warped Product Manifolds, Curvature Tensor, Pseudo-Jacobi Operator

\section{Introduction}

The Riemann curvature tensor is one of the central concepts in the mathematical field of differential geometry. It assigns a tensor to each point of a (semi-)Riemannian manifold that measures the extent to which the metric tensor is not locally isometric to that of Euclidean space. It expresses the curvature of (semi-)Riemannian. Curvature tensor is a central mathematical tool in the theory of general relativity and gravity.

The geometry of a pseudo-Riemannian manifold $(M, g)$ is the study of the curvature $R \in \otimes^{4} T^{*} M$ which is defined by the Levi-Civita connection $\nabla$. Since the whole curvature tensor is difficult to handle, the investigation usually focuses on different objects whose properties allow us to recover curvature tensor. One can for example associate to $R$ an endomorphism on tangent bundle of a manifold. In [1] P. Gilkey studied geometric properties of natural operators 
defined by the Riemann curvature tensor and Osserman proposed in [2] a characterization of Riemannian rank 1-symmetric spaces in terms of the spectrum of the Jacobi operator. Many other central works have been done by Ivanova, Stanilov, Videv and Szabo [3] [4] [5] [6] [7].

On lightlike geometry of hypersurfaces, C. Atindogbe and K. L. Duggal have studied Pseudo-Jacobi operators and considered Osserman conditions [8], and in [9], the authors introduced the notion of $r$-lightlike Osserman submanifolds.

Let $(M, g)$ be a semi-Riemannian manifold $(M, g)$ and $p \in M$. An element $R \in \otimes^{4} T_{p}^{*} M$ is said to be an algebraic curvature tensor on $T_{p} M$ if $R$ has the following symmetries:

$$
\begin{gathered}
R(X, Y, Z, W)=R(Z, W, X, Y)=-R(Y, X, Z, W) \\
R(X, Y, Z, W)+R(Y, Z, X, W)+R(Z, X, Y, W)=0 \quad \forall X, Y, Z, W \in T_{p} M .
\end{gathered}
$$

The Riemannian curvature tensor of a Levi-Civita connection is algebraic on $T_{p} M$ for all $p \in M$. If $R$ is an algebraic curvature tensor on $T_{p} M$, the associated Jacobi operator $J_{R}(X)$ with respect to $X \in T_{p} M$ is the self-adjoint linear map on $T_{p} M$ characterized by the identity

$$
g\left(J_{R}(X) Y, Z\right)=R(Y, X, X, Z) \forall Y, Z \in T_{p} M .
$$

It is obvious that $\forall c \in \mathbb{R}^{\star}, J_{R}(c X)=c^{2} J_{R}(X)$ and the domain of $J_{R}(X)$ is the unit pseudo-sphere of unit timelike or unit spacelike vectors

$$
S^{ \pm}(M):=\left\{X \in T_{p} M: g(X, X)= \pm 1\right\} .
$$

Due to the algebraic properties (1) and (2) of the curvature, we have $J_{R}(X) X=0$ and $g\left(J_{R}(X) Y, X\right)=0$. Then, the Jacobi operator naturally reduces to the endomorphism $J_{R}(X): X^{\perp} \rightarrow X^{\perp}$.

The Riemannian curvature tensor $R$ of a semi-Riemannian manifold $(M, g)$ is said to be a spacelike (resp. timelike) Osserman tensor on $T_{p} M$ if the spectrum spec $\left(J_{R}\right)$ is constant on $S_{p}^{+}(M)$ (resp. $S_{p}^{-}(M)$ ). If this is the case at each $p \in M$, we say that $(M, g)$ is pointwise Osserman semi-Riemannian manifold.

Motivated by the recent works on lightlike geometry, we consider in this paper lightlike warped product (sub-)manifolds and examine Osserman conditions depending on geometric properties of the factors.

In Section 2, we present background materials of lightlike geometry. In Section 3 we define lightlike warped product Osserman (definition 3.2) and present some important results of our research (Theorem 2, Theorem 3, Theorem 4). Section 4 is concerned with an example given in the neutral semi-Riemannian space $R_{3}^{6}$..

\section{Preliminaries}

Let $(\bar{M}, \bar{g})$ be a $(m+k)$-dimensional semi-Riemannian manifold of constant index $q$ such that $1 \leq q<m+k$ and $(M, g)$ be an $m$-dimensional submanifold of $\bar{M}$. We assume that both $m$ and $k$ are $\geq 1$. At each point $p \in M$, 


$$
T_{p} M^{\perp}=\left\{X \in T_{p} \bar{M}, \bar{g}_{p}(X, Y)=0 \forall Y \in T_{p} M\right\}
$$

is the normal space at $p$. In case $\bar{g}_{p}$ is non-degenerate on $T_{p} M$, both $T_{p} M$ and $T_{p} M^{\perp}$ are non-degenerate and we have $T_{p} M \cap T_{p} M^{\perp}=\{0\}$. If the mapping

$$
\operatorname{Rad}(T M): p \in M \mapsto \operatorname{Rad}\left(T_{p} M\right)=T_{p} M \cap T_{p} M^{\perp}
$$

is a smooth distribution with constant rank $r>0, M$ is said to be lightlike (or null) submanifold of $\bar{M}$, with nullity degree $r$. This mapping is called the radical distribution on $M$. Any complementary (and hence orthogonal) distribution of $\operatorname{Rad}(T M)$ in $T M$ is called a screen distribution. For a fixed screen distribution on $M$, the tangent bundle splits as

$$
T M=\operatorname{Rad}(T M) \oplus_{\text {Orth }} S(T M)
$$

where $\oplus_{\text {orth }}$ is the orthogonal direct sum.

A screen transversal vector bundle $S\left(T M^{\perp}\right)$ on $M$ is any (semi-Riemannian) complementary vector bundle of $\operatorname{Rad}(T M)$ in $T M^{\perp}$. It is obvious that both $S\left(T M^{\perp}\right)$ and $S(T M)^{\perp}$ is non-degenerate with respect to $\bar{g}$ and

$$
S\left(T M^{\perp}\right) \subset S(T M)^{\perp} .
$$

A null submanifold $M$ with nullity degree $r$ equipped with a screen distribution $S(T M)$ and a screen transversal vector bundle $S\left(T M^{\perp}\right)$ is denoted $\left(M, S(T M), S\left(T M^{\perp}\right)\right)$. It is said to be

- $r$-lightlike if $r<\min (m, k)$;

- coisotropic if $r=k<m$ (hence $S\left(T M^{\perp}\right)=\{0\}$ );

- isotropic if $r=m<k$, (hence $S(T M)=\{0\}$ );

- totally null if $r=m=k$, (hence $S(T M)=\{0\}=S\left(T M^{\perp}\right)$ ).

For any local frame $\left\{\xi_{i}\right\}$ of $\operatorname{Rad}(T M)$, there exists a local frame $\left\{N_{i}\right\}$ of sections with values in the orthogonal complement of $S\left(T M^{\perp}\right)$ in $S(T M)^{\perp}$ such that

$$
g\left(\xi_{i}, N_{j}\right)=\delta_{i j}, \quad g\left(N_{i}, N_{j}\right)=0,
$$

and it follows that there exists a lightlike transversal vector bundle $\operatorname{lt}(T M)$ locally spanned by $\left\{N_{i}\right\}$.

If we denote by $\operatorname{tr}(T M)$ a (not orthogonal) complementary vector bundle to $T M$ in $\left.T \bar{M}\right|_{M}$, the following relations hold

$$
\begin{gathered}
\operatorname{tr}(T M)=\operatorname{ltr}(T M) \oplus_{\text {Orth }} S\left(T M^{\perp}\right) \\
\left.T \bar{M}\right|_{M}=T M \oplus \operatorname{tr}(T M)=S(T M) \oplus_{\text {Orth }}(\operatorname{RadTM} \oplus \operatorname{ltr}(T M)) \oplus_{\text {Orth }} S\left(T M^{\perp}\right) .
\end{gathered}
$$

The Gauss and Weingarten formulas are

$$
\begin{aligned}
& \bar{\nabla}_{X} Y=\nabla_{X} Y+h(X, Y), \\
& \bar{\nabla}_{X} V=-A_{V} X+\nabla_{X}^{t} V,
\end{aligned}
$$

$\forall X, Y \in \Gamma(T M), V \in \Gamma(\operatorname{tr}(T M))$. The components $\nabla_{X} Y$ and $-A_{V} X$ belong to $\Gamma(T M), h(X, Y)$ and $\nabla_{X}^{t} V$ to $\Gamma(\operatorname{tr}(T M)) . \nabla$ and $\nabla^{t}$ are linear con- 
nections on $T M$ and the vector bundle $\operatorname{tr}(T M)$ respectively. According to the decomposition (8), let $L$ and $S$ denote the projection morphisms of $\operatorname{tr}(T M)$ onto $\operatorname{ltr}(T M)$ and $S\left(T M^{\perp}\right)$ respectively, $h^{l}=L \circ h, h^{s}=S \circ h$ where $\circ$ is the composition law, $D_{X}^{l} V=L\left(\nabla_{X}^{t} V\right), D_{X}^{s} V=S\left(\nabla_{X}^{t} V\right)$. The transformations $D^{l}$ and $D^{s}$ do not define linear connections but Otsuki connections on $\operatorname{tr}(T M)$ with respect to the vector bundle morphisms $L$ and $S$. Then,

$$
\begin{gathered}
\bar{\nabla}_{X} Y=\nabla_{X} Y+h^{l}(X, Y)+h^{s}(X, Y) \\
\bar{\nabla}_{X} N=-A_{N} X+D_{X}^{l} N+D^{s}(X, N) \\
\bar{\nabla}_{X} W=-A_{W} X+\nabla_{X}^{s} W+D^{l}(X, W) \\
\forall X, Y \in \Gamma(T M), N \in \Gamma(\operatorname{ltr}(M)) \text { and } W \in \Gamma\left(S\left(T M^{\perp}\right)\right) .
\end{gathered}
$$

Since $\bar{\nabla}$ is a metric connection, using (12)-(14) we have

$$
\begin{gathered}
\bar{g}\left(h^{s}(X, Y), W\right)+\bar{g}\left(Y, D^{l}(X, W)\right)=g\left(A_{w} X, Y\right) \\
\bar{g}\left(D^{s}(X, N), W\right)=\bar{g}\left(N, A_{W} X\right) .
\end{gathered}
$$

Let $P$ the projection morphism of $T M$ onto $S(T M)$. Using the decomposition (6) we get

$$
\begin{gathered}
\nabla_{X} Y=\nabla_{X}^{*} P Y+h^{*}(X, P Y) \\
\nabla_{X} \xi=-A_{\xi}^{*} X+\nabla_{X}^{*} \xi
\end{gathered}
$$

$\forall X, Y \in \Gamma(T M), \xi \in \Gamma(\operatorname{Rad}(T M))$ and $\nabla^{*}$ is a metric connection on $S(T M)$.

It follows from (17) and (18) that

$$
\begin{aligned}
& \bar{g}\left(h^{l}(X, P Y)\right)=g\left(A_{\xi}^{*} X, P Y\right) \\
& \bar{g}\left(h^{*}(X, P Y), N\right)=g\left(A_{N} X, P Y\right) \\
& \bar{g}\left(h^{l}(X, \xi), \xi\right)=0, A_{\xi}^{*} \xi=0 .
\end{aligned}
$$

Let $\bar{R}$ and $R$ denote the Riemannian curvature tensors on $\bar{M}$ and $M$ respectively. The Gauss equation is given by

$$
\begin{aligned}
\bar{R}(X, Y) Z= & R(X, Y) Z+A_{h^{l}(X, Z)} Y-A_{h^{l}(Y, Z)} X+A_{h^{s}(X, Z)} Y-A_{h^{s}(Y, Z)} X \\
& +\left(\nabla_{X} h^{l}\right)(Y, Z)-\left(\nabla_{Y} h^{l}\right)(X, Z)+D^{l}\left(X, h^{s}(Y, Z)\right) \\
& -D^{l}\left(Y, h^{s}(X, Z)\right)+\left(\nabla_{X} h^{s}\right)(Y, Z)-\left(\nabla_{Y} h^{s}\right)(X, Z) \\
& +D^{s}\left(X, h^{l}(Y, Z)\right)-D^{s}\left(Y, h^{s}(X, Z)\right)
\end{aligned}
$$

$\forall X, Y, Z, U \in \Gamma(T M)$. Therefore

$$
\begin{aligned}
\bar{R}(X, Y, Z, P U)= & R(X, Y, Z, P U)+\bar{g}\left(h^{*}(Y, P U), h^{l}(X, Z)\right) \\
& -\bar{g}\left(h^{*}(X, P U), h^{l}(Y, Z)\right)+\bar{g}\left(h^{s}(Y, P U), h^{s}(X, Z)\right) \\
& -\bar{g}\left(h^{s}(X, P U), h^{s}(Y, Z)\right) .
\end{aligned}
$$

Definition 2.1 ([10]). A lightlike submanifold $(M, g)$ of a semi-Riemannian 
manifold $(\bar{M}, \bar{g})$ is totally umbilical in $\bar{M}$ if there is a smooth transversal vector field $\boldsymbol{H} \in \Gamma(\operatorname{tr}(T M))$ on $M$ called the transversal curvature vector field of $M$ such that, for all $X, Y \in \Gamma(T M)$

$$
h(X, Y)=g(X, Y) \boldsymbol{H} .
$$

Using (10) and (12) it is easy to see that $M$ is totally umbilical if and only if on each coordinate neighbourhood $\mathcal{U}$ there exist smooth vector fields $H^{l} \in \Gamma(\operatorname{ltr}(T M))$ and $H^{s} \in \Gamma\left(S\left(T M^{\perp}\right)\right)$ such that

$$
\begin{gathered}
h^{l}(X, Y)=g(X, Y) H^{l}, D^{l}(X, W)=0 \\
h^{s}(X, Y)=g(X, Y) H^{s}, \forall X, Y \in \Gamma(T M), W \in \Gamma\left(S\left(T M^{\perp}\right)\right) .
\end{gathered}
$$

Definition 2.2 ([10]). Let $(M, g)$ be a $r$-lightlike (i.e. $r<\min \{m, k\})$ or a coisotropic m-dimensional submanifold of a $(m+k)$-dimensional semi-Riemannian manifold $(\bar{M}, \bar{g})$. We say that the screen distribution $S(T M)$ is totally umbilical if for any section $N$ of $\operatorname{ltr}(T M)$ on a coordinate neighbourhood $\mathcal{U} \subset M$, there exists a smooth function $\lambda$ on $\mathcal{U}$ such that

$$
\bar{g}\left(h^{*}(X, P Y), N\right)=\lambda g(X, P Y), \forall X, Y \in \Gamma\left(\left.T M\right|_{\mathcal{U}}\right) .
$$

Definition 2.3. A coisotropic submanifold $(M, g)$ of a semi-Riemannian manifold $(\bar{M}, \bar{g})$ is screen locally conformal if the local second fundamental forms of the screen distribution $S(T M)$ are related with the local second fundamental form of $M$ as follows:

$$
h_{i}^{*}(X, P Y)=\phi_{i} h_{i}^{l}(X, P Y), \forall X, Y \in \Gamma(T M)
$$

where $\phi_{i}$ is a conformal smooth function on a coordinate neighbourhood $\mathcal{U}$ in $M$. In particular, we say that $M$ is screen homothetic if $\phi_{i}$ is a non-zero constant.

Let $\left(M^{m}, g\right)$ be a null submanifold with nullity degree $r$ of a semi-Riemannian manifold $\left(\bar{M}^{m+k} \bar{g}\right),\left(\xi_{i}\right)_{i}$ and $\left(N_{i}\right)_{i}$ local frames of $\Gamma(\operatorname{Rad}(T M))$ and $\Gamma(\operatorname{ltr}(T M))$ respectively satisfying $\bar{g}\left(\xi_{i}, N_{i}\right)=\delta_{i j}$. Consider the 1 -forms $\eta_{i}, i=1, \cdots, r$ metrically equivalent to the $N_{i}$ i.e. $\eta_{i}()=.\bar{g}\left(N_{i}, \cdot\right)$. Then, each tangent vector field $X$ has the splitting,

$$
X=P X+\sum_{i=1}^{r} \eta_{i}(X) \xi_{i} .
$$

From now on, we assume that the frames $\left(\xi_{i}\right)_{i}$ and $\left(N_{i}\right)_{i}$ are globally defined on $M$. Consider the $\Gamma\left(T^{*} M\right)$ values mapping $b_{g}$ defined on $\Gamma(T M)$ by

$$
X^{b_{g}}:=b_{g}(X)=i_{X} g+\sum_{i=1}^{r} \eta_{i}(X) \eta_{i}
$$

where $i_{X}$ denotes the interior product with respect to $X$. The mapping $b_{g}$ is an isomorphisme of $\Gamma(T M)$ onto $\Gamma\left(T^{*} M\right)$ and we let $b_{g}$ denote its reverse mapping. $\forall X \in \Gamma(T M)$ (resp $\left.\omega \in \Gamma\left(T^{*} M\right)\right), X^{b_{g}}$ is called the dual 1-form of $X$ and $\omega^{b_{g}}$ the dual field of $\omega$ with respect to the pair of frames $\left(\xi_{i}\right)_{i}$ and 
$\left(N_{i}\right)_{i}$. Define a $(0,2)$-tensor $\tilde{g}$ on $M$ by

$$
\tilde{g}=g+\sum_{i=1}^{r} \eta_{i} \otimes \eta_{i}
$$

i.e.

$$
\tilde{g}(X, Y)=X^{b_{g}}(Y)=g(X, Y)+\sum_{i=1}^{r} \eta_{i}(X) \eta_{i}(Y) \forall X, Y \in \Gamma(T M) .
$$

It is straightforward to check that $\tilde{g}$ defines a non-degenerate metric on $M$ and that for $r=0$ it coincides with $g$. The $(0,2)$-tensor $\tilde{g}^{-1}$ is called the pseudo-inverse of $g$. Let $\left\{\xi_{1}, \cdots, \xi_{r}, e_{r+1}, \cdots, e_{m}\right\}$ be a quasi-orthonormal field of frames on $M$ with respect to the decomposition (6). Using (30) we have

$$
\tilde{g}\left(\xi_{i}, \xi_{j}\right)=\delta_{i j}, 1 \leq i, j \leq r \text { and }
$$

$\tilde{g}\left(e_{i}, e_{j}\right)=g_{i j}, r+1 \leq i, j \leq m$ since $\tilde{g}$ conincides with $g$ on $S(T M)$.

Definition 2.4. Let $\left(M_{0}, g_{0}\right)$ and $\left(M_{i}, g_{i}\right)(i=1,2, \cdots, l)$ be semi-Riemannian and $\left.\rho_{i}: M_{0} \rightarrow\right] 0,+\infty[$ be positive smooth functions. The multiply warped product $M=M_{0} \times{ }_{\rho_{1}} M_{1} \times{ }_{\rho_{2}} M_{2} \times \cdots \times{ }_{\rho_{l}} M_{l}$ is the product manifold $M_{0} \times M_{1} \times M_{2} \times \cdots \times M_{l}$ furnished with the metric tensor

$$
g=\pi_{0}^{*}\left(g_{0}\right)+\sum_{i=1}^{l}\left(\rho_{i} \circ \pi_{0}\right)^{2} \pi_{i}^{*}\left(g_{i}\right)
$$

where $\pi_{0}: M \rightarrow M_{0}, \pi_{i}: M \rightarrow M_{i}, i=1, \cdots, l$ are the projection morphisms. The functions $\rho_{i}$ are called the warping functions and $\left(M_{0}, g_{0}\right)$ the base manifold of the multiply warped product. Each $\left(M_{i}, g_{i}\right), i=1, \cdots, l$ is called a fiber manifold.

- If $l=1$ then we obtain a singly warped product

- If $\rho_{i}=1$ for $i=1, \cdots, l$ then we have a multiple product manifold.

- If all $\left(M_{i}, g_{i}\right), i=0,1, \cdots, l$ are Riemanniann then $(M, g)$ is also a Riemannian multiply warped product manifold. $(M, g)$ is Lorentzian multiply warped product if $\left(M_{i}, g_{i}\right), i=1, \cdots, l$ are Riemannian and either $\left(M_{0}, g_{0}\right)$ is Lorentzian or a one-dimensional manifold with a negative definite metric $-d t^{2}$.

- $(M, g)$ is lightlike with nullity degree $r$ if $\left(M_{0}, g_{0}\right)$ is degenerate with $\operatorname{Rad}\left(T M_{0}\right)$ of rank $r$. Rad (TM) still has rank $r$ and all screen structure on $M$ has dimension $s_{0}+\sum_{i=1}^{m} \operatorname{dim}\left(M_{i}\right)$ where $s_{0}$ is the dimension of any screen structure on $M_{0}$.

Proposition 1. [11] On $\left(M_{1} \times_{\rho} M_{2}, g\right)$, if $X, Y \in \Gamma\left(T M_{1}\right) ; V, W \in \Gamma\left(T M_{2}\right)$, then

1) $\nabla_{X} Y \in \Gamma\left(T M_{1}\right)$ is the lift of $\nabla_{X}^{1} Y$,

2) $\nabla_{X} V=\nabla_{V} X=\frac{X(\rho)}{\rho} V$,

3) $\left(\nabla_{V} W\right)_{V}$ is the lift of $\nabla_{V}^{2} W$,

4) $\left(\nabla_{V} W\right)_{\mathcal{H}}=\alpha(V, W)=-\frac{\langle V, W\rangle}{\rho} \operatorname{grad} \rho$. 
Corollary 1 . The leaves $M_{1} \times\{q\}$ of the warped product are totally geodesic; the fibers $\{p\} \times M_{2}$ are totally umbilical.

\section{Lightlike Warped Product Geometry and Osserman Conditions}

As it is well known, Jacobi operators are associated to algebraic curvature maps (tensors). But contrary to non-lightlike manifolds, the induced Riemann curvature tensor of a lightlike submanifold $\left(M, g, S(T M), S\left(T M^{\perp}\right)\right)$ is not an algebraic curvature map in general as it can be seen from (23). In case this requirement is satisfied, the pair of screens $\left(S(T M), S\left(T M^{\perp}\right)\right)$ is said to be admissible.

In semi-Riemannian case, the relation (3) characterizes the Jacobi operator $J_{R}($.$) associated to an algebraic curvature tensor R \in \otimes^{4} T_{p}^{*} M, \forall p \in M$. For $X \in S_{p}^{+}(M) \quad\left(\right.$ or $\left.X \in S_{p}^{-}(M)\right), Y, Z \in T_{p} M$, we have

$$
J_{R}(X) Y=R(Y, X, X, .)^{\#} \text {, }
$$

that is

$$
\left(J_{R}(X) Y\right)^{b}(Z)=R(Y, X, X, Z) .
$$

For degenerate warped product setting, we consider the associated non-degenerate metric $\tilde{g}$ defined by (30) of a lightlike warped product metric. We denote by $b_{\tilde{g}}$ and $\#_{\tilde{g}}$ the natural isomorphisms with respect to $\tilde{g}$. The equivalent relation of (3) is given by

$$
\tilde{g}\left(J_{R}(X) Y, Z\right)=R(Y, X, X, Z) .
$$

Definition 3.1. Let $\left(M, g, S(T M), S\left(T M^{\perp}\right)\right)$ be a lightlike warped product submanifold of a semi-Riemannian manifold $(\bar{M}, \bar{g}), \quad p \in M, X \in S_{p}^{+}(M)$ (or $X \in S_{p}^{-}(M)$ ) and $R \in \otimes^{4} T_{p}^{*} M$ an algebraic curvature tensor on $T_{p} M . A$ pseudo-Jacobi operator associated to $R$ with respect to $X$ is the self-adjoint linear map $J_{R}(X)$ on $T_{p} M$ defined by

$$
J_{R}(X) Y=R(Y, X, X, .)^{\#_{\tilde{g}}}
$$

or equivalentently

$$
\left(J_{R}(X) Y\right)^{b_{\tilde{g}}}(Z)=R(Y, X, X, Z) .
$$

Definition 3.2. A lightlike warped product submanifold $(M, g)$ of a semi-Riemannian manifold $(\bar{M}, \bar{g})$ is called spacelike (resp. timelike) Osserman at $p \in M$ if for each admissible pair of screens $\left\{S(T M), S\left(T M^{\perp}\right)\right\}$ and associate induced Riemann curvature $R$, the characteristic polynomial of $J_{R}(X)$ is independent of $X \in S_{p}^{+}(M)$ (resp. $X \in S_{p}^{-}(M)$ ). If this is the case for each $p \in M$, then $(M, g)$ is called pointwise Osserman. If in addition there is no dependence with respect to $p \in M$ then $(M, g)$ is said to be globally Osserman.

Theorem 2. Let $\left(N_{1}, g_{1}\right)$ and $\left(N_{2}, g_{2}\right)$ be a totally lightlike manifold and a conformally Osserman semi-Riemannian manifold respectively. Let $f$ be an iso- 
metric immersion of $(N, g)$ in a semi-Riemannian space form $(M(c), \bar{g})$ where $N=N_{1} \times_{\rho} N_{2}$ and $g=g_{1} \oplus \rho^{2} g_{2}$. Then $(N, g)$ is a conformally Osserman Lightlike warped product submanifold of $(M(c), \bar{g})$.

Proof. Let $R^{1}, R^{2}$ and $R$ be the Riemannian curvature tensors of $N_{1}, N_{2}$ and $N$ respectively. $\left(N_{1}, g_{1}\right)$ being totally degenerate, the Riemannian curvature tensor $R^{1}$ and its Weyl tensor vanish identically. Moreover $N_{1}$ is conformally Osserman. By Theorem 5 in [12], $R$ is an algebraic curvature tensor. If we restrict our study on the product $\left(N_{1} \times N_{2}, g_{1} \oplus g_{2}\right)$, it is obvious that $N$ is a conformally Osserman manifold. The lightlike warped product metric $g$ belongs to the conformal class of $\frac{1}{\rho^{2}} g_{1} \oplus g_{2}$ which is conformally Osserman lightlike product submanifold. Since the Weyl tensor is invariant in the conformal class of a metric, we conclude that $(N, g)$ is a conformally Osserman lightlike warped product.

From definition 2.2, it is obvious that if a screen distribution $S(T M)$ is totally umbilical, the bilinear form $h^{*}$ is symmetric on $S(T M)$. By theorem 2.5 in [13] p.161, $S(T M)$ is integrable and by theorem 5.3 in [10], if the ambiant space is of constant sectional curvature, the induced Ricci tensor is symmetric.

Due to Proposition 2 in [12], we establish the following two results for coisotropic warped product $\left(N_{1} \times{ }_{\rho} N_{2}, g_{1} \oplus \rho^{2} g_{2}\right)$ with $N_{1}$ totally degenerate isometrically immersed in a semi-Riemannian space form $(M(c), \bar{g})$.

Theorem 3. Let $f:\left(N=N_{1} \times_{\rho} N_{2}, g_{1} \oplus \rho^{2} g_{2}\right) \rightarrow(M(c), \bar{g})$ be a coisotropic isometric immersion of a warped product of a totally lightlike manifold $N_{1}$ and a semi-Riemannian manifold $N_{2}$ in a semi-Riemannian space form $M(c)$ that is screen conformal. Then the associated Ricci tensor is symetric and $N$ is locally Einstein. Also, $\mathrm{N}$ is pointwise Osserman.

Proof. From (22), the induced Riemannian curvature tensor is

$$
R(X, Y) Z=c\{g(Y, Z) X-g(X, Z) Y\}+A_{h^{l}(Y, Z)} X-A_{h^{l}(X, Z)} Y
$$

Using (20) and (27), for all $X, Y, Z, T \in \Gamma(T M)$, we get

$$
\begin{aligned}
R(X, Y, Z, T)= & c\{g(Y, Z) g(X, T)-g(X, Z) g(Y, T)\} \\
& +\bar{g}\left(h^{*}(X, P T), h^{l}(Y, Z)\right)-\bar{g}\left(h^{*}(Y, P T), g(X, Z)\right) \\
= & c\{g(Y, Z) g(X, T)-g(X, Z) g(Y, T)\} \\
& +\sum_{i=1}^{r} r\left(\frac{\xi_{i}(\rho)}{\rho}\right)^{2} \phi_{i} g(X, T) g(Y, Z) \\
& -\sum_{i=1}^{r} r\left(\frac{\xi_{i}(\rho)}{\rho}\right)^{2} \phi_{i} g(Y, T) g(X, Z) \\
= & {\left[c+\sum_{i=1}^{r} r\left(\frac{\xi_{i}(\rho)}{\rho}\right)^{2} \phi_{i}\right][g(Y, Z) g(X, T)-g(X, Z) g(Y, T)] . }
\end{aligned}
$$

Let $\left\{\xi_{1}, \cdots, \xi_{r}, e_{r+1}^{\prime}=\frac{e_{r+1}}{\rho}, \cdots, e_{m}^{\prime}=\frac{e_{m}}{\rho}\right\}$ be a quasi-orthonormal field of frames 
on $\left.T N\right|_{\mathcal{U}}$ where $\left\{e_{r+1}, \cdots, e_{m}\right\}$ is a orthonormal field of frames on $T N_{2}$. Then, for all $X, Y \in \Gamma(T M)$ we compute the induced Ricci curvator tensor as follow

$$
\begin{aligned}
\operatorname{Ric}(X, Y) & =\sum_{i=r+1}^{m} \epsilon_{i} g\left(R\left(e_{i}^{\prime}, X\right) Y, e_{i}^{\prime}\right)+\sum_{i=1}^{r} \bar{g}\left(R\left(\xi_{i}, X\right) Y, N_{i}\right) \\
& =\left[c+\sum_{i=1}^{r} r\left(\frac{\xi_{i}(\rho)}{\rho}\right)^{2} \phi_{i}\right][(m-r) g(X, Y)-g(X, Y)+r g(X, Y)] \\
& =\left\{\left[c+\sum_{i=1}^{r} r\left(\frac{\xi_{i}(\rho)}{\rho}\right)^{2} \phi_{i}\right](m-1)\right\} g(X, Y) .
\end{aligned}
$$

Thus the induced Ricci curvature tensor is symmetric and $N$ is locally Einstein. Consider $X \in S_{p}^{ \pm}(N), p \in N$ and $Y, Z \in X^{\perp}$. By (37) we have

$$
\tilde{g}\left(J_{R}(X) Y, Z\right)=R(Y, X, X, Z)=\left\{c+\sum_{i=1}^{r} r\left(\frac{\xi_{i}(\rho)}{\rho}\right)^{2} \phi_{i}\right\} g(X, X) g(Y, Z) .
$$

The pseudo-Jacobi operator $J_{R}(X)$ is given by

$$
J_{R}(X)= \pm\left\{c+\sum_{i=1}^{r} r\left(\frac{\xi_{i}(\rho)}{\rho}\right)^{2} \phi_{i}\right\} P Y
$$

and its characteristic polynomial is

$$
P_{\gamma}\left(J_{R}(X)\right)=(-1)^{r} \gamma^{r}\left\{ \pm\left[c+\sum_{i=1}^{r} r\left(\frac{\xi_{i}(\rho)}{\rho}\right)^{2} \phi_{i}\right]-\gamma\right\}^{m-r} .
$$

Therefore $N$ is pointwise Osserman.

From Proposition 2, theorem 5 in [12] and Theorem 4.3 in [9], we proved the following result that characterizes any screen distribution of a coisotropic warped product of a semi-Riemannian space form with the first factor totally null. This case consists of a class of null warped products that is Einstein and pointwise Osserman.

Theorem 4. Let $\left(N=N_{1} \times_{\rho} N_{2}, g_{1} \oplus \rho^{2} g_{2}\right)$, be a coisotropic warped product submanifold of a semi-Riemannian space forme $(M(c), \bar{g})$ where $N_{1}$ is totally degenerate. Then any screen distribution is admissible and totally umbilical on $N$. In addition $N$ is locally Einstein and pointwise Osserman.

\section{Example}

Let $\bar{M}=\left(\mathbb{R}_{3}^{6}, \bar{g}\right)$ be a semi-Riemannian manifold, where $\mathbb{R}_{3}^{6}$ is semi-Euclidean space of signature $(3,3)$ with respect to the canonical basis $\left(\partial x_{1}, \partial x_{2}, \cdots, \partial x_{6}\right)$. Let $M$ be submanifold of $\mathbb{R}_{3}^{6}$ given by

$$
\begin{gathered}
x^{1}=v^{1}, \quad x^{2}=v^{2} \\
x^{3}=v^{1} \cos v^{3} \sinh v^{4} \\
x^{4}=v^{1} \cos v^{3} \cosh v^{4} \\
x^{5}=v^{2}, v ; x^{6}=v^{1} \sin v^{3}
\end{gathered}
$$


where $v^{3} \in \mathbb{R}-\left\{k \frac{\pi}{2}, k \in \mathbb{Z}\right\}$.

Then $T M$ is spanned by

$$
\begin{gathered}
X_{1}=\partial x_{1}+\cos v^{3} \sinh v^{4} \partial x_{3}+\cos v^{3} \cosh v^{4} \partial x_{4}+\sin v^{3} \partial x_{6} \\
X_{2}=\partial x_{2}+\partial x_{5} \\
X_{3}=-v^{1} \sin v^{3} \sinh v^{4} \partial x_{3}-v^{1} \sin v^{3} \cosh v^{4} \partial x_{4}+v^{1} \cos v^{3} \partial x_{6} \\
X_{4}=v^{1} \cos v^{3} \cosh v^{4} \partial x_{3}+v^{1} \cos v^{3} \sinh v^{4} \partial x_{4}
\end{gathered}
$$

$T M^{\perp}=\operatorname{span}\left\{X_{1}, X_{2}\right\}$. Thus $M$ is a 2-lightlike submanifold of $\mathbb{R}_{3}^{6}$ with $\operatorname{RadTM}=\operatorname{span}\left\{X_{1}, X_{2}\right\}$. Choose $S(T M)=\operatorname{span}\left\{X_{3}, X_{4}\right\}$, we construct two null vectors

$$
\begin{gathered}
N_{1}=\frac{1}{2}\left\{-\partial x_{1}+\cos v^{3} \sinh v^{4} \partial x_{3}+\cos v^{3} \cosh v^{4} \partial x_{4}+\sin v^{3} \partial x_{6}\right\} \\
N_{2}=\frac{1}{2}\left\{-\partial x_{2}+\partial x_{5}\right\} .
\end{gathered}
$$

Since rank RadTM equals codimension of $M$, we conclude that $M$ is a coisotropic submanifold of $\bar{M}$. By direct calculations, RadTM and $S(T M)$ are integrable in $M$. The induced metric tensor $d s^{2}$ on $M$ is given by

$$
\mathrm{d} s^{2}=\left(\frac{\partial x^{\alpha}}{\partial v^{i}}\right)^{2} \bar{g}_{\alpha \alpha}\left(\mathrm{d} v^{i}\right)^{2}, \alpha=1, \cdots, 6 ; i=1, \cdots, 4
$$

and we get

$$
\mathrm{d} s^{2}=\left(v^{1}\right)^{2}\left(\mathrm{~d} v_{3}^{2}-\cos ^{2} v^{3} \mathrm{~d} v_{4}^{2}\right)
$$

It is obvious that $M$ is a coisotropic warped product submanifold of $\mathbb{R}_{3}^{6}$ with warping function $\rho=v^{1}$. Using (12) and (17) we obtain

$$
\begin{gathered}
h^{l}\left(X, X_{1}\right)=h^{l}\left(X, X_{2}\right)=h^{l}\left(X_{3}, X_{4}\right)=0, \forall X \in \Gamma(T M) \\
h^{l}\left(X_{3}, X_{3}\right)=-v^{1} N_{1}, h^{l}\left(X_{4}, X_{4}\right)=v^{1} \cos ^{2} v^{3} N_{1} . \\
h^{*}\left(X, X_{1}\right)=h^{*}\left(X, X_{2}\right)=h^{*}\left(X_{3}, X_{4}\right)=0, \forall X \in \Gamma(T M) \\
h^{*}\left(X_{3}, X_{3}\right)=-\frac{1}{2} v^{1} X_{1}, h^{*}\left(X_{4}, X_{4}\right)=\frac{1}{2} v^{1} \cos ^{2} v^{3} X_{1} .
\end{gathered}
$$

From (38), we have

$$
h^{l}(X, Y)=g(X, Y) H^{l} ; H^{l}=-\frac{N_{1}}{v^{1}} \forall X, Y \in \Gamma(T M)
$$

and we conclude that $M=M_{1} \times_{v^{1}} M_{2}$ is a totally umbilical null warped product submanifold of $\mathbb{R}_{3}^{6}$ and it is obvious that it is mixed totally geodesic.

From (39) $\forall X, Y \in \Gamma(T M), N \in \Gamma(\operatorname{ltr}(T M))$ we have

$$
\begin{gathered}
\bar{g}\left(h^{*}(X, P Y), N\right)=\gamma g(X, P Y) ; \quad \gamma=-\frac{1}{2 v^{1}} \\
h_{i}^{*}(X, Y)=\frac{1}{2} h_{i}^{l}(X, Y)
\end{gathered}
$$


and we conclude that the screen distribution $S(T M)$ of $M$ is totally umbilical and $M$ is screen homothetic.

$M$ being coisotropic, taking into account $\bar{M}$ has constant sectional curvature $c=0$, consider (40) and (41), from (42) we get

$$
\begin{aligned}
& R(X, Y, Z, T) \\
& =\frac{1}{2\left(v^{1}\right)^{2}}\{g(Y, Z) g(X, T)-g(X, Z) g(Y, T)\} \forall X, Y, Z, T \in \Gamma(T M) .
\end{aligned}
$$

Let's consider $X \in S_{p}^{+}(M)$, (or $\left.X \in S_{p}^{-}(M)\right), \quad p \in M, Y, Z \in X^{\perp}$. Then by using (43), we have

$$
\tilde{g}\left(J_{R}(X) Y, Z\right)=R(Y, X, X, Z)=\frac{1}{2\left(v^{1}\right)^{2}} g(X, X) g(Y, Z) .
$$

The pseudo-Jacobi operator $J_{R}(X)$ is given by $J_{R}(X) Y= \pm \frac{1}{2\left(v^{1}\right)^{2}} P Y$ and its characteristic polynomial $P_{\lambda}\left(J_{R}(X)\right)$ is given by

$$
P_{\lambda}\left(J_{R}(X)\right)=\lambda^{2}\left( \pm \frac{1}{2\left(v^{1}\right)^{2}}-\lambda\right)^{4}
$$

that is independent of $X \in S_{p}^{ \pm}(M)$. Therefore, $M$ is spacelike (timelike) pointwise Osserman null warped product.

Remark. From (44), it is obvious that for a lightlike warped product manifold, to be spacelike Osserman or timelike Osserman are equivalent.

\section{Conclusion and Suggestions}

Osserman conditions on lightlike warped product manifolds have been considered in this paper. The case of lightlike warped product with the first factor totally degenerate has been explored. Especially in coisotropic case, we have proved that this class consists of Einstein and locally Osserman lightlike warped product. In perspective, we are going to extend this study to other classes of lightlike warped product in order to get later a certain characterization of lightlike warped product Osserman manifolds.

\section{Conflicts of Interest}

The authors declare no conflicts of interest regarding the publication of this paper.

\section{References}

[1] Gilkey, P. (2002) Geometric Properties of Natural Operators Defined by the Riemann Curvature Tensor. World Scientific, Singapore. https://doi.org/10.1142/4812

[2] Osserman, R. (1990) Curvature in the Eighties. The American Mathematical Monthly, 97, 731-756. https://doi.org/10.1080/00029890.1990.11995659 
[3] Ivanova, R. and Stanilov, G. (1994) A Skew-Symmetric Curvature Operator in Riemannian Geometry. Symposia Gaussiana. Conference A: Mathematics and Theoretical Physics, 391-396. https://doi.org/10.1515/9783110886726.391

[4] Stanilov, G. (2004) Higher Order Skew-Symmetric and Symmetric Curvature Operators. Comptes rendus de I Academie bulgare des Sciences, 57, 9-12.

[5] Stanilov, G. and Videv, V. (1992) On a Generalization of the Jacobi Operator in the Riemannian Geometry. Annual of Sofia University "St Kliment Ohridski”, Facurity of Mathematics and Informormatics, 86, 27-34.

[6] Stanilov, G. and Videv, V. (2004) On the Commuting of Curvature Operators. Mathematics and Education in Mathematics, Proceedings of the 33rd Spring Conference of the Union of Bulgarian Mathematicians, Sofia, 1-4 April 2004, 176-179.

[7] Szabo, Z.I. (1991) A Simple Topological Proof for the Symmetry of 2 Point Homogeneous Spaces. Inventiones Mathematicae, 106, 61-64. https://doi.org/10.1007/BF01243903

[8] Atindogbe, C. and Duggal, K.L. (2009) Pseudo-Jacobi Operators and Osserman Lightlike Hypersurfaces. Kodai Mathematical Journal, 32, 91-108.

[9] Atindogbe, C., Lungiambudila, O. and Tossa, J. (2011) Ligthlike Osserman Submanifolds of Semi-Riemannian Manifolds. Afrika Matematika, 22, 129-151.

[10] Duggal, K.L. and Jin, D.H. (2003) Totally Umbilical Lightlike Submanifolds. Kodai Mathematical Journal, 26, 49-68. https://doi.org/10.2996/kmj/1050496648

[11] O’Neill, B. (1983) Semi-Riemannian Geometry with Applications to Relativity. Academic Press, New York.

[12] Ndayirukiye, D., Nibarura, G., Karimumuryango, M. and Nibirantiza, A. (2019) Algebraicity of Induced Riemannian Curvature Tensor on Lightlike Warped Product Manifolds. Journal of Applied Mathematics and Physics, 7, 3132-3139.

[13] Duggal, K.L. and Bejancu, A. (1996) Lightlike Sub of Semi-Riemannian and Applications. Kluwer Academic Publishers, Amsterdam, 308 p.

https://doi.org/10.1007/978-94-017-2089-2 